\title{
Simulation of Droplets Collisions Using Two-Phase Entropic Lattice Boltzmann Method
}

\author{
A. Mazloomi Moqaddam ${ }^{1}$. S. S. Chikatamarla ${ }^{1}$. \\ I. V. Karlin ${ }^{1}$
}

Received: 6 July 2015 / Accepted: 9 July 2015 / Published online: 23 July 2015

C Springer Science+Business Media New York 2015

\begin{abstract}
The recently introduced entropic lattice Boltzmann model for multiphase flows (Mazloomi et al. in Phys Rev Lett 114:174502, 2015) is used to simulate binary droplet collisions. The entropy-based stabilization, together with a new polynomial equation of state, enhances performance of the model and allow us to simulate droplet collision for various Weber and Reynolds numbers and large liquid to vapor density ratio. Different types of droplet collision outcomes, namely coalescence, stretching separation and reflexive separation are recovered in a range of impact parameter for two equal sized droplets. The results demonstrated the essential role played by the surface tension, kinematic viscosity, impact parameter and relative velocity in the droplet collision dynamics leading to coalescence or separation collision outcomes. Comparison between numerical results and experiments in both coalescence and separation collisions demonstrate viability of the presented model.
\end{abstract}

Keywords Entropic lattice Boltzmann method - Two-phase flow · Droplet collision dynamics

\section{Introduction}

Study of binary droplet collisions sheds light on many applications across different scientific disciplines, from understanding cloud formation in climate theory to engineering applications such as turbine blade cooling, ink-jet printing, spray coating, and spray combustion in diesel internal combustion engines. The prediction of the collision dynamics of liquid droplets is

S. S. Chikatamarla

chikatamarla@lav.mavt.ethz.ch

A. Mazloomi Moqaddam

alim@lav.mavt.ethz.ch

I. V. Karlin

karlin@lav.mavt.ethz.ch

1 Department of Mechanical and Process Engineering, ETH Zurich, 8092 Zurich, Switzerland 
considered to be a challenging and important problem in fluid dynamics [22] and has regularly been used to benchmark various models for multiphase flows. Therefore, droplet collision has been the subject of numerous investigations, ranging from experimental, analytical to numerical studies. However, the complexity and the small scale motion encountered in such problems has mostly limited the full investigation to either experimental visualization of droplet collision images or analytical studies based on simplified models. Therefore, direct numerical simulations focusing on the collision dynamics of liquid drops are indeed very scarce [7]. Also, the numerical simulation of multiphase flows is a challenging subject due to a number of issues related to the tracking of interfaces, mass conservation of each fluid, and the treatments of large density ratio and surface tension.

Numerous experimental investigations of droplet collisions have been reported in the literature $[3,9,25]$. Most of these studies illustrated the general motion of the droplet and provided collision images without sufficient time resolution which makes it difficult to describe the intricacies of the collision dynamics for instance. Hence, a large number of investigations were performed analytically to provide information on the probability of occurrence of particular type of collision [11,35]. In general, most of the developed droplet collision models lack comprehensiveness and have not been adequately validated. Moreover, most of the commonly used droplet interaction analytical models are based upon experimental observations of the behavior of water droplets. On the other hand, experiments of Qian and Law [26] on hydrocarbon drops at high pressure show that the results can be significantly different from those of water drops at atmospheric pressure. Thus further development of numerical models can help advance the understanding of the dynamics and behavior of droplets during the collision process.

Numerical simulations can provide important details which are not easily observed experimentally, such as velocity and vorticity field in both the liquid and vapor phases. This and the superior flexibility of simulations in the choice of initial and boundary conditions as well as fluid properties make the numerical simulation an important tool for predicting the significant characteristics of fluid dynamics. For numerical simulations of binary droplet collisions, several numerical methods have been used for tracking the liquid-gas interface, including the Marker-And-Cell method, the front tracking method, level-set method, smoothed particle hydrodynamic method and volume-of-fluid method (VOF) $[1,23,34,36]$. The VOF methodology with high accuracy and low computational cost has been successfully applied by Nikolopoulos et al. [23,24] to study the binary collisions of fluid droplets, and good agreement was achieved between their simulations and the experimental results of Qian and Law [26]. Chen et al. [4,5] predicted the droplet behavior after collision using an improved VOF technique, and adaptive mesh refinement algorithm and mass transfer process. Dai and Schmidt [6] used a three-dimensional moving-mesh unstructured finite volume solver to investigate the effect of viscosity on the maximum deformation amplitude. However, these numerical methods are limited by inherent complexities of the multiphase flows such as tracking interface, change of mass and momentum across interface and bringing behavior of the large density ratios between fluids and surface tension upon interface.

Recently, the lattice Boltzmann method (LBM) became mainstream approach to incompressible and weakly compressible flows. Applications of LBM range from hydrodynamics at large Reynolds numbers to flows at a micron scale, porous media, and multiphase flows [32]. LBM solves a fully discrete kinetic equation for populations $f_{i}(\boldsymbol{x}, t)$. Populations correspond to discrete velocities $\boldsymbol{v}_{i}, i=0, \ldots, N$, which fit into a regular spatial lattice with the nodes $\boldsymbol{x}$. This enables a simple "stream along links and equilibrate at nodes "realization of the LBM algorithm. A limitation of the standard LBM is the inability [32] to attain low viscosity because of numerical instabilities. Hence, large Reynolds numbers in the standard 
LBM simulation can be achieved only by grid refinement. Recent theoretical development of the entropic lattice Boltzmann method (ELBM) $[2,14]$ alleviates this obstacle by restoring the second law of thermodynamics (Boltzmann's H-theorem). This basic physical requirement renders ELBM nonlinearly stable numerically.

Various approaches on how to treat multiphase flows in the framework of LBM have been presented (for a recent review, see e.g. [31] and references therein). Many of them followed the idea first presented by Shan and Chen (SC) [30]. The SC approach relies on a microscopic picture of multiphase flows, where an additional forcing term based on nearest neighbor interaction mimics the effect intermolecular interaction. Further development of the SC model was undertaken by many authors, in particular [29] (the multirange pseudo-potential method) and $[18,39]$ (general equations of state). Interested reader can find a concise review of the to-date status of the SC modeling of multiphase physics in [17]. The thermodynamic inconsistency of SC-type models was explained by He and Doolen in [12] and constitutes the major reason why we choose an alternative, and more intuitive approach due to Swift et al. [33] which is based on a direct forcing of the Korteweg's stress onto the lattice Boltzmann system.

With the focus on the droplet collisions, few LBM simulations can be found in the literature. Inamuro et al. [13] produced droplet collision results at Reynolds number of around 2000 and Weber number around 100 at density ratio of up to 50, employing projection method applied to the free energy model. Lycett-Brown et al. [20] developed a cascaded LBM [10] realization of the SC model (based on a formulation of the cascaded LBM by Karlin [15]). They simulated droplets collisions up to $W e=100$ and $R e=1000$, with density ratio over 100. The aforementioned approach, however inherited the problems for the SC model and is computationally intensive due to additional cost for incorporating central moments. Using SC model with a MRT scheme is carried out by Luo et al. [19] to achieve Reynolds numbers of up to approximately hundred and Weber number up to 100 at a density ratio around 50 .

In this paper, we apply the recently introduced ELBM for two-phase fluid flows [21] at both large density ratios and low viscosity so that one can simulate binary droplet collisions for various Weber and Reynolds numbers. Moreover, a thermodynamically consistent freeenergy approach without further simplifications is employed. The difficulty in the treatment of large density ratios is resolved by using a polynomial equation of state (EoS) and application of the ELBM. This resolves the limitation on the viscosity typical to most free energy based simulations reported in the literature.

The remainder of the paper is organized as follows: The ELBM for two-phase fluids [21] is reviewed in Sect. 2. Validation of the method and numerical results on the binary droplet collision are presented in Sect. 3. Finally, conclusions are drawn in Sect. 4.

\section{Numerical Method}

\subsection{Entropic Lattice Boltzmann Method for Two-Phase Fluids}

Here we use the recently proposed entropic lattice Boltzmann model for two-phase flows [21] which we briefly review. The classical capillarity theory by van der Waals [37] and Korteweg [16] is followed below. Free-energy functional is composed of a bulk free energy, $\Psi$, and an excess free energy stored at the liquid-vapor interface,

$$
G=\int\left[\Psi(\rho, T)+\frac{\kappa}{2}|\nabla \rho|^{2}\right] d \boldsymbol{x}
$$


where $\kappa$ is the surface tension coefficient, and $\rho$ is the local density. Equation (1) implies Korteweg's stress [16] (see [8,28]),

$$
\boldsymbol{P}=\left(p-\kappa \rho \nabla^{2} \rho-\frac{\kappa}{2}|\nabla \rho|^{2}\right) \boldsymbol{I}+\kappa(\nabla \rho) \otimes(\nabla \rho),
$$

where $p$ is the EoS, $p=\rho(\partial \Psi / \partial \rho)-\Psi$, and $\boldsymbol{I}$ is unit tensor.

Implementation of Korteweg's stress (2) in the lattice Boltzmann setting was first suggested by Swift et al. [33], and refined by Wagner and Li [38]. In the forcing method of implementation of multiphase models, the local flow velocity is altered by an amount $\delta \boldsymbol{u}=(\boldsymbol{F} / \rho) \delta t$, where $\delta t=1$ is the lattice time step, and $\boldsymbol{F}$ is the force related to Korteweg's stress,

$$
\boldsymbol{F}=\nabla \cdot\left(\rho c_{\mathrm{s}}^{2} \boldsymbol{I}-\boldsymbol{P}\right) .
$$

where $c_{\mathrm{s}}^{2}$ is the lattice speed of sound.

The entropic lattice Boltzmann equation is written,

$$
\begin{aligned}
& f_{i}\left(\boldsymbol{x}+\boldsymbol{v}_{i}, t+1\right)-f_{i}(\boldsymbol{x}, t)=\alpha \beta\left[f_{i}^{\mathrm{eq}}(\rho, \boldsymbol{u})-f_{i}(\boldsymbol{x}, t)\right] \\
& \quad+\left[f_{i}^{\mathrm{eq}}(\rho, \boldsymbol{u}+\delta \boldsymbol{u})-f_{i}^{\mathrm{eq}}(\rho, \boldsymbol{u})\right]
\end{aligned}
$$

where $0<\beta<1$ is a parameter related to the kinematic viscosity, $\nu=\mu / \rho=\left(\beta^{-1}-1\right) c_{\mathrm{s}}^{2} / 2$. The equilibrium $f^{\text {eq }}$ is the minimizer of the discrete entropy function $H$ under the constraints of local conservation laws of mass and momentum, $\{\rho, \rho \boldsymbol{u}\}=\sum_{i=0}^{N}\left\{1, \boldsymbol{v}_{i}\right\}\left\{f_{i}^{\mathrm{eq}}\right\}$, where

$$
H=\sum_{i=0}^{N} f_{i} \ln \left(f_{i} / W_{i}\right),
$$

with $W_{i}$ the lattice-specific weights. For the sake of computational efficiency, it suffices to use the expansion of the minimization problem to order $u^{3}$ (see also [27]),

$$
f_{i}^{\mathrm{eq}}=\rho W_{i}\left(1+\frac{v_{i \alpha} u_{\alpha}}{c_{\mathrm{s}}^{2}}+\frac{u_{\alpha} u_{\beta}}{2 c_{\mathrm{s}}^{4}}\left(v_{i \alpha} v_{i \beta}-c_{\mathrm{s}}^{2} \delta_{\alpha \beta}\right)+\frac{u_{\alpha} u_{\beta} u_{\gamma}}{6 c_{\mathrm{s}}^{6}} v_{i \gamma}\left(v_{i \alpha} v_{i \beta}-3 c_{\mathrm{s}}^{2} \delta_{\alpha \beta}\right)\right) .
$$

Key point is the parameter $\alpha$ in (4) which maintains the entropy balance in the relaxation step at each node; and is available as the non-trivial root of the equation,

$$
H\left(f+\alpha\left(f^{\mathrm{eq}}-f\right)\right)=H(f) .
$$

The last term on the right hand side of Eq. (4) is a specific realization of the forcing, the so-called exact difference method (EDM) [18]. To this end, the difference from the standard LB models for the two-phase flow is the ELBM relaxation ( $\alpha$ is computed from the entropy balance (7), as opposed to taking the fixed value $\alpha=2$ ).

\subsection{Nonideal Equation of State}

Finally, it remains to specify EoS $p$. A polynomial nonideal gas EoS of Ref. [21] is used in the present work. In order to construct the polynomial EoS, we begin with the Peng-Robinson (PR) EoS, as given by Yuan and Schaefer [39]:

$$
\begin{aligned}
p_{\mathrm{PR}}(\rho, T) & =\frac{\rho R_{G} T}{1-b_{\mathrm{PR}} \rho}-\frac{a_{\mathrm{PR}} \sigma(T) \rho^{2}}{1+2 b_{\mathrm{PR}} \rho-b_{\mathrm{PR}}^{2} \rho^{2}}, \\
\sigma(T) & =\left[1+\left(0.37464+1.54226 \omega-0.26992 \omega^{2}\right)\left(1-\sqrt{T / T_{c}}\right)\right]^{2},
\end{aligned}
$$


with $a_{\mathrm{PR}}=2 / 49, b_{\mathrm{PR}}=2 / 21, R_{G}=1$, and with the acentric factor $\omega=0.344$ corresponding to water (see [39]). The choice of the parameters corresponds to the critical temperature $T_{c}=0.0729$. Next, a different EoS is constructed as a polynomial in both density and temperature, in the form

$$
p(\rho, T)=\rho R_{G} T(1+b \rho \lambda(\rho, T))-a \rho^{2},
$$

with $a=9 / 49, b=2 / 21$ (the van der Waals parametrization, cf. [39]) and $R_{G}=1$. Furthermore, function $\lambda$ is the density-dependent collision probability [12] considered here as a polynomial in density,

$$
\lambda=\sum_{k=0}^{4} A_{k}(T) \rho^{k},
$$

and where, in turn, functions $A_{k}$ are polynomials of the temperature,

$$
A_{k}(T)=\sum_{n=0}^{m} a_{k n} T^{n} .
$$

The $5 \times(m+1)$ numerical coefficients $a_{k n}$ in (12) are defined from matching the values of the function (10) with the original PR EoS (8),

$$
p\left(\rho_{r}, T_{s}\right)=p_{\mathrm{PR}}\left(\rho_{r}, T_{s}\right), \quad r=0, \ldots, 4, \quad s=0, \ldots, m ;
$$

where the density and the temperature values are chosen as follows: (i) For each selected $T_{s}$, the fourth-order polynomial $p\left(\rho, T_{s}\right)$ matches the isotherm $p_{\mathrm{PR}}\left(\rho, T_{i}\right)$ at the minimum, the maximum, at the saturated vapor, at the saturated liquid and at the intercept (the latter three points are the Maxwell equal area rule); (ii) $(m+1)$ values of the temperature for this matching procedure [in other words, $(m+1)$ PR isotherms] are selected uniformly in the subcritical regime, between the lowest value $T_{0}=0.04486$ and the highest value $T_{m}=0.07042$. It was found that with $m=6$, the polynomial EoS reproduces well the non-polynomial PR EoS in the domain of interest. Numerical values of the coefficients in these polynomials are given in Table 1. The use of the polynomial EoS (10) instead of the PR EoS is motivated by the absence of the singularity in the former. Using the EoS (10), we were able to achieve high density ratios in the dynamic regime, and also improve the performance of free energy models.

Simulations were performed using the standard 27-velocity lattice, $c_{\mathrm{s}}^{2}=1 / 3$, with the discrete velocities and the corresponding weights as follows:

$$
\begin{gathered}
\boldsymbol{v}_{i}= \begin{cases}(0,0,0) & i=0 ; \\
( \pm 1,0,0),(0, \pm 1,0),(0,0, \pm 1) & i=1,2, \ldots, 6 \\
( \pm 1, \pm 1,0),( \pm 1,0, \pm 1),(0, \pm 1, \pm 1) & i=7,8, \ldots, 18 \\
( \pm 1, \pm 1, \pm 1) & i=19,20, \ldots, 26\end{cases} \\
W_{i}= \begin{cases}8 / 27 & i=0 ; \\
2 / 27 & i=1,2, \ldots, 6 ; \\
1 / 54 & i=7,8, \ldots, 18 \\
1 / 216 & i=19,20, \ldots, 26\end{cases}
\end{gathered}
$$

Thermodynamic consistency of the present model was validated numerically by simulating the coexistence curve as shown in Fig. 1. It can be seen that the densities of liquid and vapor phases in the simulation agree well with the values predicted by Maxwell's equal area rule. The maximal density ratio $r=\rho_{\mathrm{l}} / \rho_{\mathrm{v}}$ of liquid and vapor phases achieved in these simulations was $r>800$. 


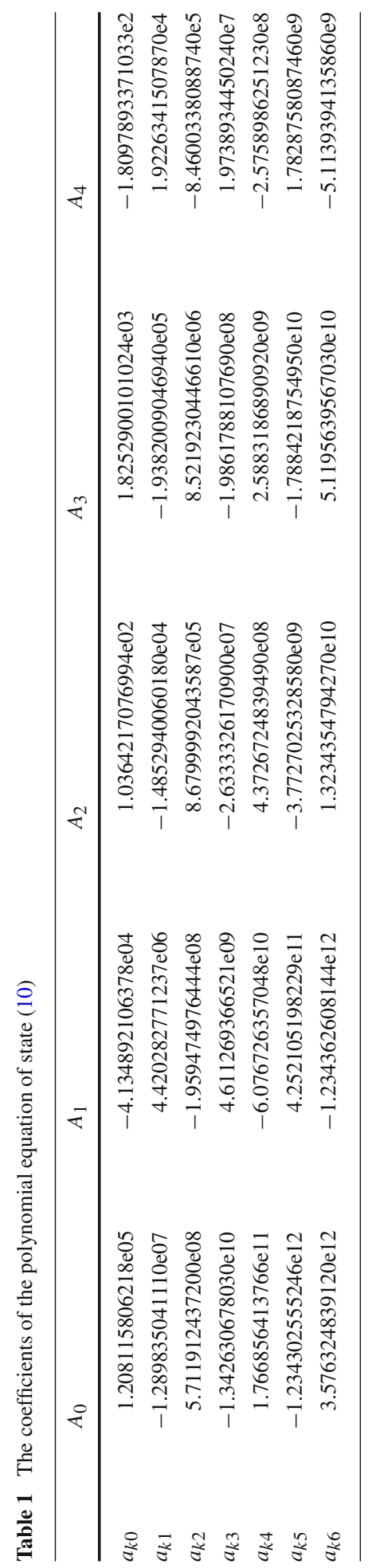


Fig. 1 The coexistence curve. Symbol ELBM simulation; line Maxwell's equal area rule

Fig. 2 Schematic representation of the binary droplet collision
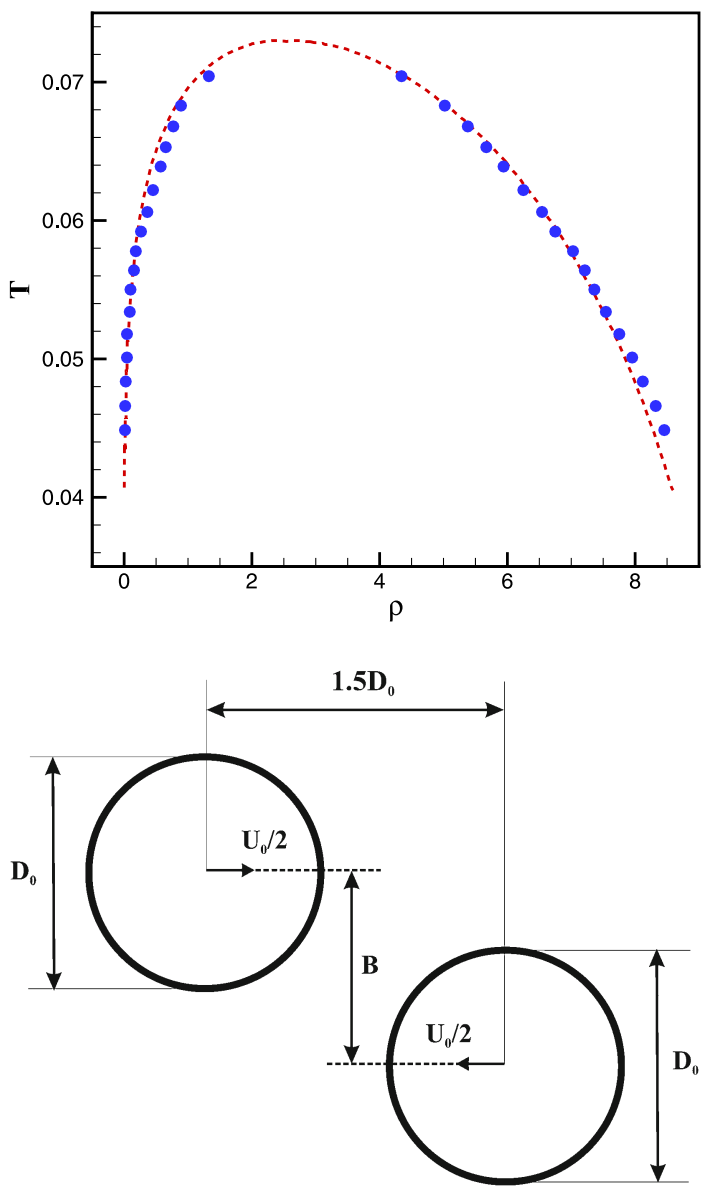

\section{Results and Discussion}

Collision setup for equal-size droplets is investigated using the above method, as sketched in Fig. 2. Two liquid droplets of diameter $D_{0}$ are initially placed $1.5 D_{0}$ apart, with the centers offset $B$, in equilibrium with the surrounding vapor. Droplets were set into motion with the initial relative velocity $U_{0}$. In general, depending on the sizes of the impinging drops, their kinetic energy, the impact parameter and the fluids properties, the outcome of the collision may differ. All these factors can be parametrized in terms of a group of dimensionless numbers; namely the Weber number (We), the Reynolds number (Re) and the impact parameter $(\chi)$,

$$
\operatorname{Re}=\frac{U_{0} D_{0}}{\nu_{1}}, \quad \mathrm{We}=\frac{\rho_{\mathrm{l}} U_{0}^{2} D_{0}}{\sigma}, \quad \chi=\frac{B}{D_{0}},
$$

where $\sigma$ is surface tension and $\rho_{1}$ and $\nu_{1}$ are density and viscosity of liquid droplet, respectively.

A collision occurs when $0 \leq \chi<1$ and $U_{0}>0$. When $B=0$ a head-on collision occurs otherwise an off-center collision happens. The material properties of the fluids used in the present simulations are $\sigma=0.353, \rho_{\mathrm{l}}=7.8, \rho_{\mathrm{v}}=0.07$ and $v_{1}=v_{\mathrm{v}}$ in range of 0.1 


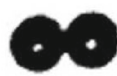

$\mathrm{t}=\mathbf{0}(\mathrm{ms})$

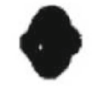

$t=0.66$

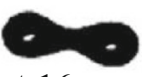

$\mathrm{t}=\mathbf{1 . 6}$

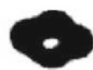

$\mathbf{t}=\mathbf{2 . 2 7}$

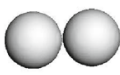

$\mathrm{t}=\mathbf{0}(\mathrm{ms})$
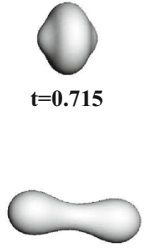

$\mathrm{t}=\mathbf{1 . 4 5}$

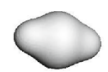

$\mathrm{t}=\mathbf{2 . 0 5}$

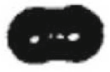

$\mathrm{t}=\mathbf{0 . 0 5}$

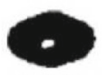

$t=0.78$

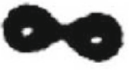

$\mathrm{t}=1.8$

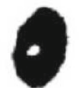

$t=2.74$

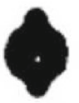

$\mathrm{t}=\mathbf{0 . 1 6}$

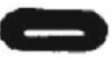

$\mathbf{t}=\mathbf{0 . 9 2}$

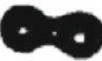

$\mathbf{t}=\mathbf{2 . 0 2}$

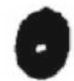

$\mathrm{t}=\mathbf{2 . 8 8}$

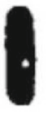

$\mathrm{t}=\mathbf{0 . 2}$

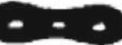

$t=1.23$

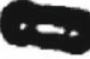

$\mathbf{t}=\mathbf{2 . 1}$

(a)
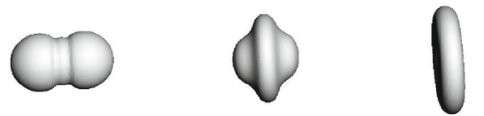

$\mathrm{t}=\mathbf{0 . 1 5 4}$

$\mathrm{t}=\mathbf{0 . 2 3 5}$
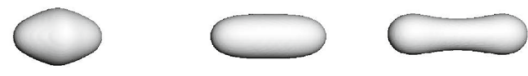

$\mathrm{t}=\mathbf{0 . 9 1 1}$

$\mathbf{t}=1.19$

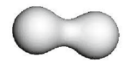

$\mathrm{t}=\mathbf{1 . 7 8}$

$\mathbf{t}=\mathbf{1 . 8 7}$

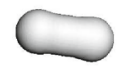

$t=1.62$
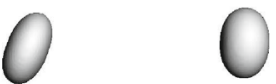

$\mathrm{t}=\mathbf{2 . 4}$

(b)

Fig. 3 Sequence of coalescence observed for binary collision of equal size droplets at $\mathrm{We}=32.8, \operatorname{Re}=210.8$, $\chi=0.08$ and $D_{0}=318 \mu \mathrm{m}$. a Experiment of Qian and Law (Ref. [26]); b simulation

to 0.01 , depending on the Reynolds numbers. Periodic boundary condition was set for all the six sides of the computational domain. At the beginning of the simulation, $t=0$, the droplets are seeded with a gap $\left(1.5 D_{0}\right)$ and a uniform velocity $U_{0} / 2$ is imposed on each of them in opposite directions without any driving external force while the surrounding vapor is initialized in a static state. The computational domain is determined according to the size of the droplets and the stretching they undergo. The following simulations, unless otherwise stated, were performed on a $8 D_{0} \times 4 D_{0} \times 4 D_{0}$ grid with an initial droplet diameter of $D_{0}=80$ grid points. In this study all the parameters and variables are given in dimensionless units 


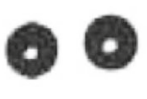

$\mathrm{t}=\mathbf{0}(\mathrm{ms})$

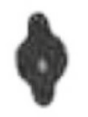

$\mathbf{t}=\mathbf{0 . 2 4}$

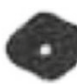

$\mathrm{t}=\mathbf{0 . 7 6}$

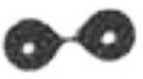

$\mathrm{t}=\mathbf{1 . 8 5}$

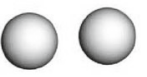

$\mathrm{t}=\mathbf{0}(\mathrm{ms})$

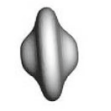

$\mathrm{t}=\mathbf{0 . 2 3}$

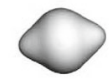

$\mathbf{t}=\mathbf{0 . 8 4 7}$

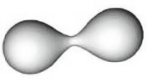

$\mathrm{t}=\mathbf{1 . 7 8}$

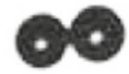

$\mathrm{t}=\mathbf{0 . 0 5}$

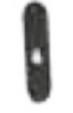

$\mathrm{t}=\mathbf{0 . 3 4}$

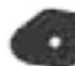

$\mathrm{t}=\mathbf{0 . 8 5}$

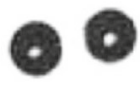

$\mathrm{t}=\mathbf{1 . 9 3}$

(a)

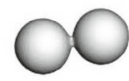

$\mathbf{t}=\mathbf{0 . 0 5 1}$

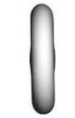

$\mathrm{t}=\mathbf{0 . 3 2 5}$

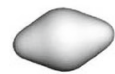

$\mathrm{t}=\mathbf{0 . 8 8 2}$

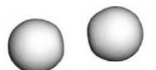

$\mathrm{t}=\mathbf{1 . 8 4}$

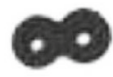

$\mathrm{t}=\mathbf{0 . 1}$

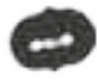

$\mathbf{t}=\mathbf{0 . 1 3}$

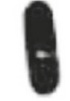

$\mathrm{t}=\mathbf{0 . 5 6}$

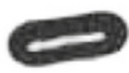

$\mathrm{t}=\mathbf{0 . 9 3}$

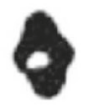

$t=0.67$

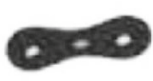

$\mathrm{t}=\mathbf{1 . 2 5}$
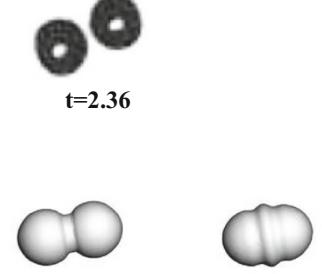

$\mathrm{t}=\mathbf{0 . 0 9 3}$

$\mathrm{t}=\mathbf{0 . 1 2 8}$

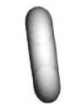

$\mathbf{t}=\mathbf{0 . 5 9 7}$

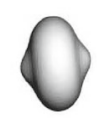

$\mathrm{t}=\mathbf{0 . 8 0 2}$

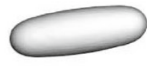

$\mathrm{t}=\mathbf{1 . 0 4 4}$

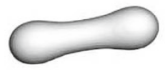

$\mathrm{t}=\mathbf{1 . 3 8}$

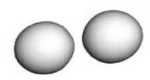

$\mathrm{t}=\mathbf{2 . 2 3}$

(b)

Fig. 4 Near head-on reflexive separation collision at $\mathrm{We}=37.2, \mathrm{Re}=228.0, \chi=0.01$ and $D_{0}=328 \mu \mathrm{m}$. a Experiment of Qian and Law (Ref. [26]); b simulation

after rescaling with the relative velocity $U_{0}$ for the velocity, with the droplet initial diameter $D_{0}$ for the length, and with $D_{0} / U_{0}$ for the time.

It is known that, depending on the Weber number and the impact parameter, different types of collision outcomes can be observed in the experiment [3]. These distinct regimes are specified as coalescence and bouncing at low to moderate We, reflexive separation for higher We and lower impact parameter, and stretching separation for both higher We and higher impact parameter. Before studying various regimes of droplet collisions, validation of the method is required. To that end, we compare the simulation results with those reported by experiments [26]. 


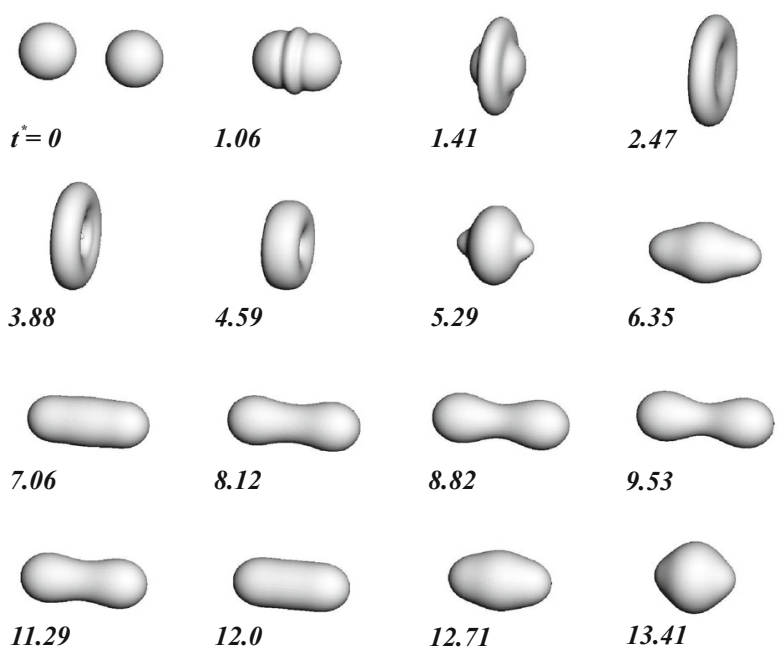

Fig. 5 Time evolution of the head-on droplet collision at $\mathrm{We}=50, \operatorname{Re}=263$ and $\chi=0.0$

The result of a near head-on collision at $W e=32.8, R e=210.8$ and $\chi=0.08$ is shown in Fig. 3, and it clearly reproduces the experiments by Qian and Law [26] also shown in the same figure. A sequence of configurations during the collision is generated from the simulation for a direct comparison with the experimentally observed shapes. To make a direct comparison, the simulation starts at the moment when the two drops are separated by the same distance as that in the experiment at $t=0$. It is evident from Fig. 3 that the process of the collision is well captured by the simulation.

Figure 4 compares the simulation results with another experimental outcome which leads to a reflexive separation for near head-on droplet collision. Direct comparison of the simulation results with experiments reveal a reasonable agreement. In both the comparisons, the droplet shapes as a function of collision time are reproduced satisfactorily. Grid size for these simulations is $150 \times 150 \times 150$ with $D_{0}=30$.

The time evolution of droplets shape at $W e=50, R e=263$ and $\chi=0$ is shown in Fig. 5. It is clearly seen from Fig. 5 that after two droplets collide head-on, a disk-like droplet is formed at $t^{*}=2.47$. Large curvature at the circumference of the disk-like droplet raises the pressure difference between the inner and the outer regions caused by surface tension effects. As a result, the disk contracts radially inwards from $t^{*}=2.47$ to $t^{*}=4.59$ and pushes the liquid outwards at $t^{*}=5.29$ from its center to form a liquid cylinder at $t^{*}=7.06$. After forming rounded ends at $t^{*}=9.53$, the liquid cylinder oscillates to become a spherical droplet. Coalescence collision is clearly the outcome of this type of collision.

Figure 6 shows the simulation results of time evolution of head-on collision at $W e=76$ and $R e=324$. Results of this simulation indicate deformation of the coalesced droplet increases as the Weber number keeps increasing. Similar to previous case, after two droplet collide, they initially form an outward spreading disk, this disk eventually contracts because of surface tension and creates a liquid cylinder which stretches out along initial coalescence axis $\left(t^{*}=6.96\right.$ to $\left.t^{*}=14.8\right)$ until the two end-drops are connected by a ligament. At $t^{*}=16.1$, the inertia overcomes the surface tension forces and this results in breaking of the ligament. Eventually, two separate droplets are seen in this type of collision which also belongs to the reflexive separation class. 


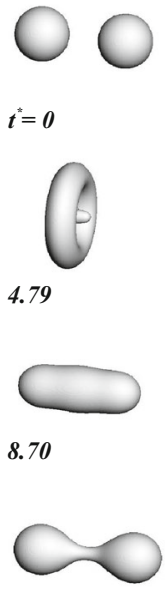

13.93

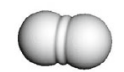

0.87

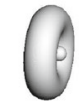

5.66

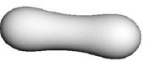

10.01

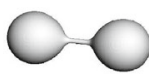

14.80

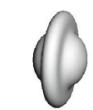

1.74

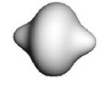

6.96

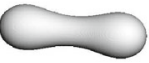

10.88

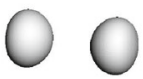

16.10
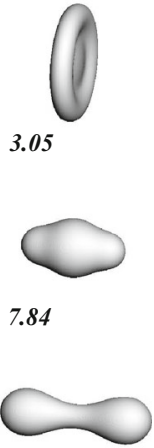

12.19

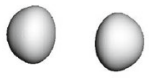

16.98

Fig. 6 Time evolution of the reflexive separation collision at $\mathrm{We}=64.3, \mathrm{Re}=324.2, \chi=0.0$

Off-center droplet collisions have been simulated for the same Weber and Reynolds number while impact parameter varied between 0.1 and 0.82 . Simulation results are shown in Fig. 7. Off-center collisions resulting in a coalescence are shown in Fig. 7a, b and c. In the range of $\chi=0.1-0.3$, the flow gained during the coalescence includes radial flows and axial flows which result in spherical rear parts of the colliding drops. The radial flow expands the colliding drops in the radial direction resulting in a thin liquid sheet call lamella inside the torus. Because of the extra axial momentum caused by high Weber number, the lamella ruptures and results in a small liquid mass in the center and the surrounding torus. One can see in Fig. 7a to $\mathrm{c}$ that the torus contracts and connects with the small droplet in middle under surface tension effects, and ends up in a full coalescence.

If the Weber and the Reynolds number are kept the same as in the above cases but the impact parameter is increased, the regime of stretching separation is observed, Fig. 7d, e and $\mathrm{f}$. In the regime of stretching separation, the collision may produce one or more satellite droplets between the larger trailing (primary) drops. The capillary-wave instability and the end-pinching are two conjectured reasons for the liquid ligament breakup [3,26]. Figure $7 \mathrm{~d}$ shows that the two ending droplets are pinched off from the liquid cylinder with a liquid thread left in the middle. The liquid thread oscillates and eventually becomes a satellite droplet.

The collision regime of stretching separation in which lobe-shaped drops are formed after collision, are shown in Fig. 7e and f. One can observe a strong rotational motion during droplet formation caused by large impact parameter in Fig. 7e. Owing to this rotation, a slender liquid cylinder is generated in between and then disintegrates into smaller drops. The typical interface width observed in our simulations varies from two to five grid points depending on the radius of curvature and the dynamics of the droplet. If the impact parameter become larger (Fig. 7f), only a small portion of the droplets touch each other and the remaining portion of the droplets tend to move in the direction of their initial velocity. Therefore, a long liquid filament is generated between the trailing and leading droplets before eventually breaking up into a very small liquid filament in the middle as shown in Fig. $7 \mathrm{f}$. 


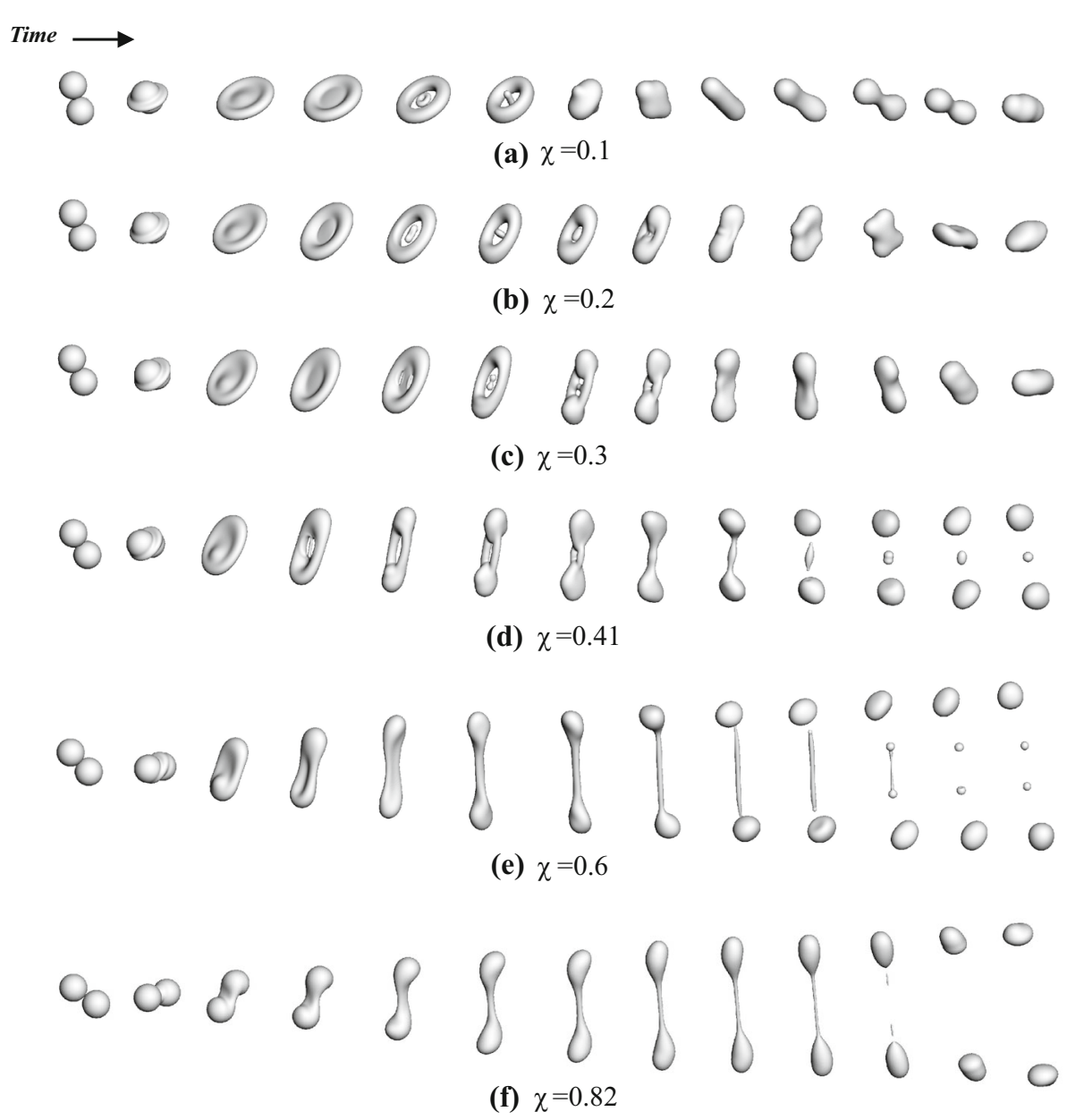

Fig. 7 Process of the binary droplet collisions for various impact parameter at $\mathrm{We}=76$ and $\mathrm{Re}=262$

\section{Conclusion}

We used the entropic lattice Boltzmann method for two-phase flows [21] to simulate threedimensional binary droplet collisions for various Weber numbers and impact parameters. Low viscosity is achieved due to the use of entropic approach while the large density ratios are attained by employing polynomial EoS. Although the density ratio of the liquid and vapor phases are not as high as in the experiments (typically of the order 1000) it is clear that the vapor phase has minimal influence on the liquid phase (due to density ratio of around 100) and this is sufficient for us to recover all the experimental results. The free energy model together with the entropic approach was shown to be a reliable tool for predicting droplet dynamics. This fills in the gap in the literature where most of the droplet dynamics simulations so far were performed using the Shan-Chen model because of the drawbacks of the previous formulations of the free energy model. The method [21] was applied to equal size binary droplet collisions, and all three types of expected collision regimes, coalescence, reflexive separation and stretching separation were clearly observed. Good agreement between the 
simulation results and experimental observations was demonstrated. A number of simulations were performed to determine the influence of Weber number and impact parameter. Further simulations by varying Reynolds numbers, Weber numbers and the sizes of the colliding droplets are underway and will be reported elsewhere. Simulation of a droplet impacting on a solid surface are planned for the near future.

Acknowledgments This work was supported by the European Research Council (ERC) Advanced Grant No. 291094-ELBM and the ETH Research Grant ETH35-12-2. Computational resources at the Swiss National Super Computing Center, CSCS, were provided under the Grant S492 .

\section{References}

1. Acevedo-Malavé, A., García-Sucre, M.: 3D coalescence collision of liquid drops using smoothed particle hydrodynamics. INTECH Publ. 5, 85-106 (2011)

2. Ansumali, S., Karlin, I.V., Öttinger, H.C.: Minimal entropic kinetic models for hydrodynamics. Europhys. Lett. 63(6), 798 (2003)

3. Ashgriz, N., Poo, J.Y.: Coalescence and separation in binary collisions of liquid drops. J. Fluid Mech. 221, 183-204 (1990)

4. Chen, X.D., Ma, D.J., Khare, P., Yang, V.: Energy and mass transfer during binary droplet collision. In: 49th AIAA Aerospace Sciences Meeting Including the New Horizons Forum and Aerospace Exposition, p. 771. Orlando (2011)

5. Chen, X.D., Ma, D.J., Yang, V.: Collision outcome and mass transfer of unequal-sized droplet collision. In: Proceedings of 50th AIAA Aerospace Sciences Meeting Including the New Horizons Forum and Aerospace Exposition (2012)

6. Dai, M., Schmidt, D.P.: Numerical simulation of head-on droplet collision: effect of viscosity on maximum deformation. Phys. Fluids 17(4), 041701 (2005)

7. Driessen, T., Jeurissen, R.: A regularised one-dimensional drop formation and coalescence model using a total variation diminishing (TVD) scheme on a single Eulerian grid. Int. J. Comput. Fluid Dyn. 25(6), 333-343 (2011)

8. Evans, R.: The nature of the liquid-vapour interface and other topics in the statistical mechanics of non-uniform, classical fluids. Adv. Phys. 28, 143-200 (1979). doi:10.1080/00018737900101365

9. Gao, T.C., Chen, R.H., Pu, J.Y., Lin, T.H.: Collision between an ethanol drop and a water drop. Exp. Fluids 38(6), 731-738 (2005)

10. Geier, M.C., Greiner, A., Korvink, J.G.: Cascaded digital lattice Boltzmann automata for high Reynolds number flow. Phys. Rev. E 73, 066705 (2006)

11. Georjon, T.L., Reitz, R.D.: A drop-shattering collision model for multidimensional spray computations. At. Sprays 9(3), 231 (1999)

12. He, X., Doolen, G.D.: Thermodynamic foundations of kinetic theory and lattice Boltzmann models for multiphase flows. J. Stat. Phys. 107(1-2), 309-328 (2002)

13. Inamuro, T., Tajima, S., Ogino, F.: Lattice Boltzmann simulation of droplet collision dynamics. Int. J. Heat Mass Transf. 47(21), 4649-4657 (2004)

14. Karlin, I.V., Ferrante, A., Öttinger, H.C.: Perfect entropy functions of the lattice Boltzmann method. Europhys. Lett. 47(2), 182 (1999)

15. Karlin, I.V., Lycett-Brown, D., Luo, K.H.: Enhanced lattice Bhatnagar-Gross-Krook method for fluid dynamics simulation. arXiv:1107.3309 (cond-mat.stat-mech) (2011)

16. Korteweg, D.J.: Sur la forme que prennent les equations du mouvements des fluides si lon tient compte des forces capillaires causes par des variations de densite. Arch. Neerl. Sci. Exact. Nat. Ser. II(6), 1-24 (1901)

17. Krüger, T., Frijters, S., Günther, F., Kaoui, B., Harting, J.: Numerical simulations of complex fluid-fluid interface dynamics. Eur. Phys. J. Spec. Top. 222, 177-198 (2013)

18. Kupershtokh, A.L., Medvedev, D.A., Karpov, D.I.: On equations of state in a lattice Boltzmann method. Comput. Math. Appl. 58(5), 965-974 (2009)

19. Luo, K.H., Xia, J., Monaco, E.: Multiscale modeling of multiphase flow with complex interactions. J. Multiscale Model. 1(01), 125-156 (2009)

20. Lycett-Brown, D., Luo, K.H., Liu, R., Lv, P.: Binary droplet collision simulations by a multiphase cascaded lattice Boltzmann method. Phys. Fluids 26(2), 023303 (2014) 
21. Mazloomi, M.A., Chikatamarla, S.S., Karlin, I.V.: Entropic lattice Boltzman method for multiphase flows. Phys. Rev. Lett. 114, 174502 (2015)

22. Nijdam, J.J., Guo, B., Fletcher, D.F., Langrish, T.A.G.: Challenges of simulating droplet coalescence within a spray. Dry. Technol. 22(6), 1463-1488 (2004)

23. Nikolopoulos, N., Nikas, K.S., Bergeles, G.: A numerical investigation of central binary collision of droplets. Comput. Fluids 38(6), 1191-1202 (2009)

24. Nikolopoulos, N., Strotos, G., Nikas, K.S., Bergeles, G.: The effect of Weber number on the central binary collision outcome between unequal-sized droplets. Int. J. Heat Mass Transf. 55(7), 2137-2150 (2012)

25. Orme, M.: Experiments on droplet collisions, bounce, coalescence and disruption. Prog. Energy Combust. Sci. 23(1), 65-79 (1997)

26. Qian, J., Law, C.K.: Regimes of coalescence and separation in droplet collision. J. Fluid Mech. 331, 59-80 (1997)

27. Qian, Y.H., Zhou, Y.: Complete Galilean-invariant lattice BGK models for the Navier-Stokes equation. Europhys. Lett. 42(4), 359-364 (1998)

28. Rowlinson, J.R., Widom, B.: Molecular Theory of Capillarity. Clarendon Press, Oxford (1982)

29. Sbragaglia, M., Benzi, R., Biferale, L., Succi, S., Sugiyama, K., Toschi, F.: Generalized lattice boltzmann method with multirange pseudopotential. Phys. Rev. E 75(2), 026702 (2007)

30. Shan, X.W., Chen, H.D.: Lattice Boltzmann model for simulating flows with multiple phases and components. Phys. Rev. E 47(3), 1815 (1993)

31. Siebert, D.N., Philippi, P.C., Mattila, K.K.: Consistent lattice Boltzmann equations for phase transitions. Phys. Rev. E 90, 053310 (2014)

32. Succi, S.: The Lattice Boltzmann Equation. Oxford University Press, Oxford (2001)

33. Swift, M.R., Osborn, W.R., Yeomans, J.M.: Lattice Boltzmann simulation of nonideal fluids. Phys. Rev. Lett. 75(5), 830 (1995)

34. Tanguy, S., Berlemont, A.: Application of a level set method for simulation of droplet collisions. Int. J. Multiph. Flow 31(9), 1015-1035 (2005)

35. Tennison, P.J., Georjon, T.L., Farrell, P.V., Reitz, R.D.: An experimental and numerical study of sprays from a common rail injection system for use in an HSDI diesel engine. Technical report, SAE Technical Paper (1998)

36. Tryggvason, G., Bunner, B., Esmaeeli, A., Juric, D., Al-Rawahi, N., Tauber, W., Han, J., Nas, S., Jan, Y.J.: A front-tracking method for the computations of multiphase flow. J. Comput. Phys. 169(2), 708-759 (2001)

37. van der Waals, J.D.: hermodynamische theorie der capillariteit in de onderstelling van continue dichtheidsverandering. Verhand. Kon. Akad. V Wetensch. Amst. (Sect. 1) 1(8), 1-54 (1893)

38. Wagner, A.J., Li, Q.: Investigation of Galilean invariance of multi-phase lattice Boltzmann methods. Phys. A 362(1), 105-110 (2006)

39. Yuan, P., Schaefer, L.: Equations of state in a lattice Boltzmann model. Phys. Fluids 18(4), 042101 (2006) 